\title{
A Delay Sensitive Feedback Control Data Aggregation Approach in Wireless Sensor Network*
}

\author{
Peng Shao-liang, Li Shan-shan, Peng Yu-xing, Zhu Pei-dong, and Xiao Nong \\ School of Computer, National University of Defense Technology, Changsha, China \\ \{pengshaoliang, ssli, pyx, pdz, xn\}@nudt.edu.cn
}

\begin{abstract}
In wireless sensor networks (WSN) real-time data delivery is a challenging issue, especially when bursty events happen and many emergent packets appear. The cluster-based data aggregation has emerged as a basic and general technique in sensor networks. However, there is a trade-off issue between aggregation waiting (AW) delay and aggregation accuracy. Therefore, we propose a Delay Sensitive Feedback Control (DSFC) model to solve the trade-off issue. Our goal is to decrease the AW delay on the condition of meeting the desired aggregation accuracy for emergent packets when emergent event occurs. Simulation results verify the performance of DSFC, and demonstrate their attraction.
\end{abstract}

Keywords: Wireless Sensor Networks, Real-Time, Feedback Control.

\section{Introduction}

Wireless sensor networks can be used for many mission-critical applications such as target tracking in battlefields, nuclear leak alert, habitat temperature monitoring in forests and hazardous environment exploration [1]. Although these applications for sensor network remain diverse, one commonality they all share is the need for a timely delivery of sensory data. Without a real-time communication service, applications cannot react to the changes of the environment quickly enough to be effective. Therefore, in this paper we propose a comprehensive mechanism to meet real-time constraint of packets generated in crisis states.

Data aggregation has emerged as a basic tenet in sensor networks. The key idea is to combine the data coming from different sensors - eliminating redundancy, minimizing the number of transmissions and thus saving energy. Data aggregation can also provide a rich, multi-dimensional view of the environment being monitored [2]. However, nowadays many works on data aggregation are based on cluster topology [3]. All vicinal clustering members aggregate data at cluster head. Clustering-based aggregation has high energy efficiency due to intra-cluster data redundancy.

Although clustering benefits from data aggregation, which may not be the only reason for using clusters. Clustering makes the system scalable. Instead of having a

\footnotetext{
Supported by the National Natural Science Foundation of China (Grant No. 60433040), the National Basic Research Program of China under Grant No 2002CB312105 (973), and the National Basic Research Program of China under Grant No 2006CB303000 (973).
} 
centralized control over thousands of nodes, or having a distributed protocol that operates over thousands of nodes, it is better to organize nodes into smaller clusters, and assign the responsibility of MAC and routing in each cluster to a single cluster head node. So we propose a bursty real-time mechanism which is cluster-based.

However, there are problems for cluster-based data aggregation: higher latency and less accuracy, especially when a lot of emergent packets are generated within a short period. We try to find the optimal point for the trade-off between aggregation delay and accuracy. That is to decrease delay as much as possible within the accuracy bound. In this paper, a distinguished feedback control model is proposed to adaptively aggregate data in a delay sensitive manner while not damage the gathering accuracy. Our goal is to decrease the AW delay on the condition of meeting the desired aggregation accuracy for emergent packets when emergent event occurs.

The rest of this paper is organized as follows. Section 2 briefly describes some related work. In Section 3, we discuss the issues of delay/accuracy trade-off and propose a Delay Sensitive Feedback Control (DSFC) model to solve this problem. Several simulations described in Section 4 verify the obtained theoretical results. The paper ends with conclusion and directions for the future in Section 5.

\section{Related Work}

Interactions between sensor networks and the physical world require real-time services to play an important role. Several real-time methods have been proposed for sensor and ad hoc networks. SPEED [4] protocol is designed to provide soft end-toend deadline guarantees for real-time packets in sensor networks. MM-SPEED [5] extends SPEED to support different delivery velocities and levels of reliability.

However, previous approaches are efficient only when most traffic requires low reporting rates, which is not the case for many sensor networks especially with bursty events. Also, it is not adaptive to dynamics of data collection. Besides the real-time protocol design, several researches approaches focus on the delay analysis for data aggregation. In [6], Athanassios et al. only focus on data aggregation: an energyaccuracy trade-off, but they can not mention aggregation delay. However, all the prior work has been mainly concerned with the benefits of aggregation and has only superficially acknowledged the delay/accuracy trade-off present.

A hierarchical network organization will not only facilitate data aggregation, but will facilitate the formation of uniform, robust backbones, and give an opportunity for nodes to sleep while not sensing or transmitting. Many clustering protocols have been investigated [7]. However, prior work has rarely been concerned with the delay of aggregation. In order to build an E2E quick path for packets of emergent events, we will put forward a bursty real-time mechanism which is ground on clustering.

The E2E delay of emergent packet is crucial when bursty events happen. The delay is mainly composed of (i) intra-cluster data aggregation (ii) inter-cluster routing. We will take into account the delay of intra-cluster data aggregation because the latter is routing issue, which has been investigated in many literatures. 


\section{Delay Sensitive Feedback Control in Intra-cluster Data Aggregation}

In this Section, we first briefly describe priority based packets classification and scheduling method because latter some mechanisms are based on it. Then, in Section 3.2, we analyze the delay due to data aggregation. Finally, in Section 3.3 we discuss the issues of delay/accuracy trade-off and propose a Delay Sensitive Feedback Control (DSFC) model to solve it.

\subsection{Priority Based Packets Classification and Scheduling}

Considering the characteristic of bursty events in wireless sensor networks, we will borrow the idea of QoS from Internet which has been proposed using two different methods -the Differentiated services (DiffServ) and the Integrated services (IntServ). We can classify the packets into two classes: emergent packet and regular packet, according to the semantic characteristics of packets. Packets classification is implemented by setting a bit in the TOS field. Cluster head assigns the TDMA slots for each node in the region according to the application of every source node, creates a TDMA schedule for its nodes and broadcasts it every round aggregation. TDMA also can be modified to allocated more and earlier slots to emergent packets.

\subsection{Delay Due to Data Aggregation}

Although data aggregation results in fewer transmissions, there is a tradeoff - potentially greater delay because data from different sources may have to be held back at a cluster head in order to be aggregated with data coming from other sources. Fewer transmissions should be achieved at the cost of delay, especially when bursty events happen and many emergent packets appear.

Generally there are three factors affecting the delay of intra-cluster data aggregation: (i)Aggregation Routing (AR), (ii) Aggregation Function (AF), (iii) Aggregation Waiting (AW). Earlier data from different sources may have to wait at a cluster head in order to be aggregated with subsequent data coming from other sources. The problem of AW delay will be aggravated especially when emergent events occur. The delay of AW is the most important bottleneck of data aggregation. In this paper, we will place emphasis on the issue of AW delay and consider how to deal with the accuracy/aggregation tradeoff.

\subsection{Trade-Off Between AW Delay and Aggregation Accuracy}

\subsubsection{Problem Statement}

In order to addressing the delay bottleneck of aggregation waiting, we must take into account the loss of aggregation accuracy. However, many nodes in the same cluster are located at the close geographical area, their gatherings are correlated and accordingly some accuracy loss is acceptable. We can consider to aggregate partial data to reduce the AW delay on the condition of meeting the desired aggregation accuracy. Therefore, there is a trade-off issue between AW delay and aggregation accuracy. 
The trade-off issue can be regarded as a problem of Multiobjective Optimization. AW delay and aggregation accuracy are two main objects but they come into conflict with each other. Consequently, we use Optimization Method to solve this trade-off problem. Optimization Method is one of the most effective and prompt means to deal with the trade-off issue. The AW delay of emergent packet is crucial when bursty events happen, so we will aim at the goal of reducing AW delay. In detail, we will put forward a Delay Sensitive Feedback Control (DSFC) model [8], which can decrease the AW delay on the condition of meeting the desired aggregation accuracy.

\subsubsection{Delay Sensitive Feedback Control Model}

We assume an ideal TDMA strategy used as our intra-cluster MAC protocol. Every source node will ask for sending packets to cluster head at a certain reporting rate before every round aggregation. Cluster head can execute a centric control every round according to the request of every source node, and assign the TDMA slots for each intra-cluster node. Each cluster head then use a Priority based Packet Scheduling (discussed in section 3.2) strategy based on TDMA to allocate time slots. Thus, emergent packets can obtain more and earlier time slots. Each source node sends data to its cluster head according to the specified TDMA schedule.

In this section, we present a Delay Sensitive Feedback Control (DSFC) model to solve the problems on delay/accuracy trade-off. We can use the DSFC model to adaptively aggregate partial data to reduce the AW delay on the condition of meeting the desired aggregation accuracy. We begin with the following definitions.

Definition1. Aggregation Scale (AS): The ratio of emergent packets arrived at cluster head to total produced emergent packets in a round of data aggregation. The calculation of AS is as followed:

$$
\operatorname{AS}(i)=\frac{N_{\text {arrived }}(i)}{N_{\text {total }}(i)}, \quad i \text { is round number, } i=1,2,3,4 \ldots .
$$

$\mathrm{N}_{\text {total }}(\mathrm{i})$ is the total number of produced emergent packets in the ith round aggregation. $\mathrm{N}_{\text {arrived }}(\mathrm{i})$ is the factual number of emergent packets that arrive at cluster head and need to be aggregated in the ith round. Obviously, $\mathrm{AS} \leq 1 . \mathrm{N}_{\text {total }}$ (i) can be calculated by cluster head because all source nodes must request to send packets at a certain reporting rate when the source nodes sense an emergent event. Cluster head can execute a centric control every round according to the request of every source node. $\mathrm{AS}(\mathrm{i})$ is used to control the aggregation accuracy and AW delay. Figure 1 shows the feedback controller, and it can control the parameter of Aggregation Function (AF). The concrete definition of AF is specific to the application of WSN, and can be expressed as $\mathrm{AF}(\mathrm{i}, \mathrm{AS}(\mathrm{i}))$. For example, $\mathrm{AF}(\mathrm{i}, 1)$ means that cluster head carry out full data aggregation until all data of source node reach cluster head. The aggregation accuracy of full data aggregation is the highest but the AW delay is the longest. 
Definition2. Error (E): The error of partial data aggregation. It can be calculated as followed:

$$
\mathrm{E}(\mathrm{i})=|\mathrm{AF}(\mathrm{i}, 1)-\mathrm{AF}(\mathrm{i}, \mathrm{AS}(\mathrm{i}))|
$$

$\mathrm{E}(\mathrm{i})$ is the difference between partial aggregation and full aggregation which means of data aggregation accuracy at $\mathrm{i}$ round. It can be used to adjust the AS (i+1) at (i+1) round aggregation. The Closed-loop Feedback Control model is illustrated as equation (3):

$$
\mathrm{AS}(\mathrm{i}+1)=\mathrm{AS}(\mathrm{i})+\alpha\left(\mathrm{E}(\mathrm{i})-\mathrm{E}_{0}\right)
$$

$\alpha$ is the controller coefficient and can be well approached by some off-line experiments. $\mathrm{E}_{0}$ is the minimal error that is related to specific applications of WSN.

The DSFC architecture is shown in figure 1:

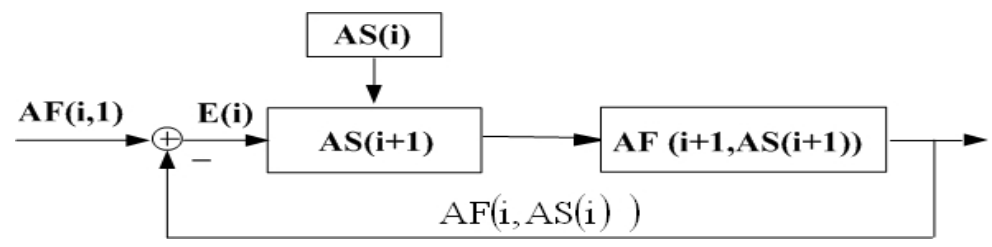

Fig. 1. The Architecture of DSFC Model

Our goal is transmit the emergent packets as fast as possible when emergent events occur. Emergent packets are partially aggregated in the proportion of AS( $i+1)$. The DSFC can adaptively decrease the AW delay at the cost of accuracy. We can continuously reduce the AS, namely $\mathrm{AS}(\mathrm{i}+1)$, as long as the aggregation accuracy meets the requirement of applications. The DSFC system tends to be steady until $\mathrm{E}(\mathrm{i})$ is close to ${ }^{E_{0}}$.

The total time of the ith round data aggregation AT(i) is composed of three parts, which is illustrated as equation (4):

$$
\text { A T (i) }=\mathrm{S}+\mathrm{C}+\sum_{\mathrm{j}=1}^{\mathrm{N} \text { arived }} \mathrm{T}_{\mathrm{j}}
$$

S: The synchronization time of intra-cluster nodes

$\mathrm{C}$ : The intercommunion time that cluster head executes a centric control and harmonization before the source data arrive at cluster head. The course includes source node requesting phase and cluster head assigning time slots phase.

$\mathrm{T}_{\mathrm{j}}$ : The time slot of packet $\mathrm{j}$. $\mathrm{T}_{\mathrm{j}}$ is assigned by cluster head.

\subsubsection{Stable State}

Under the adjustment of DSFC, the system eventually converges at a stable state. In order to further decrease the AW delay and full aggregation overhead, full aggregation 
is unnecessary in every aggregation round. Partial data aggregation can be executed with a fixed AS for a period of time.

\section{Performance Evaluation}

\subsection{Simulation Settings}

We simulate DSFC and EPCR using GloMoSim [9], a scalable discrete-event simulator developed by UCLA. This software provides a high fidelity simulation for wireless communication with detailed propagation, radio and MAC layers. Table 1 illustrates the detailed configuration for our simulation. The communication parameters are chosen in reference to the Berkeley mote specification.

Table 1. Simulation settings

\begin{tabular}{|l|l|}
\hline Routing & Shortest Path \\
\hline MAC Layer & TSMA (Intra-Cluster), 802.11 (Inter-Cluster) \\
\hline Radio Layer & RADIO-ACCNOISE \\
\hline Propagation Model & TWO-RAY \\
\hline Bandwidth & $200 \mathrm{~Kb} / \mathrm{s}$ \\
\hline Payload Size & 32 Byte \\
\hline TERRAIN & $(300 \mathrm{~m}, 300 \mathrm{~m})$ \\
\hline Node Number & 300 \\
\hline Radio Range & $30 \sim 50 \mathrm{~m}$ \\
\hline Clustering Method & HEED [10] \\
\hline Cluster Radius & $25 \mathrm{~m}$ \\
\hline
\end{tabular}

We use a simulation model based on GlomoSim and conduct extensive experiments to evaluate its performance. To make dense network with a strong connectivity and have more choice to construct cluster and paths, we deploy 300 nodes with a radio range of $30 \mathrm{~m}$ to $50 \mathrm{~m}$. A channel error rate of $10 \%$ is uniformly distributed around the network (With max ARQ of 802.11).Our works is not based on a certain routing protocol, so we select the Shortest Path routing protocol and long-hop standpoint. In our evaluation, we present the following set of results: (1) DSFC model to adaptively control Aggregation Scale, (2) AW delay and error under different scenarios, (3) aggregation communication energy consumption. All experiments are repeated 16 times with different random seeds.

\subsection{Performance of DSFC}

First, we examine the performance of intra-cluster DSFC model. In figure 2 we present the DSFC control model to adaptively aggregate partial emergent packets to reduce the AW delay at the three different scenarios. DSFC can effectively and directly control the AF to converge at a certain value according to $\mathrm{E}(\mathrm{i})$ and the data correlation. Figure 3 illustrates the AW delay of different scenarios. The emergent 


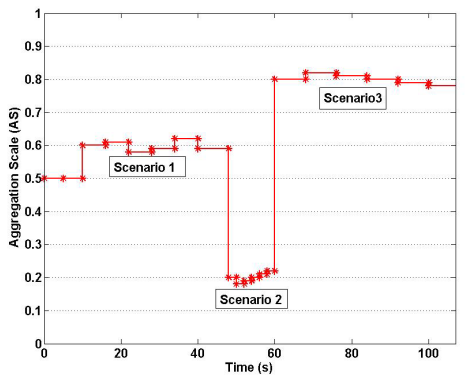

Fig. 2. Control process of DSFC model

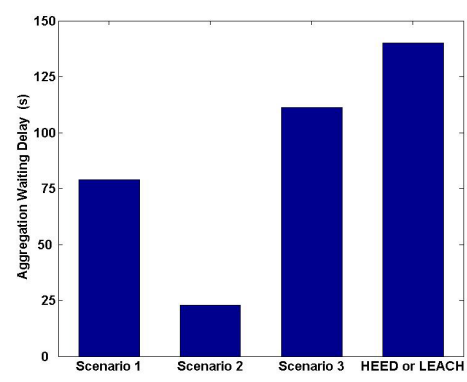

Fig. 3. AW delay of Different Scenarios

packets data aggregation based clustering generally aggregate all intra-cluster data. So, cluster head has to wait for a long time in order to be aggregated all data coming from other sources. We can see clearly in Figure 4 the AF delay of HEED and LEACH [10] is longer than that in scenario1, 2, and 3.

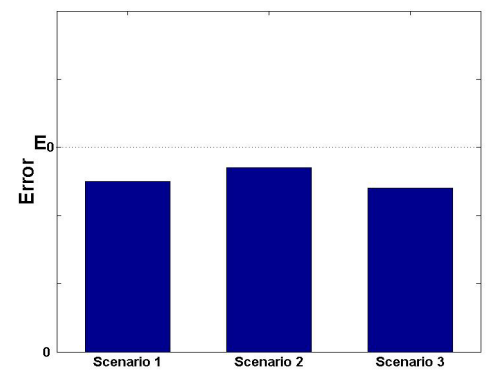

Fig. 4. Error of Different Scenarios

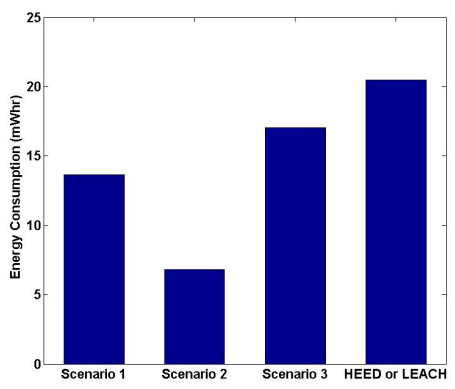

Fig. 5. Intra-cluster Energy Consumption

Figure 4 shows the error of partial data aggregation under different scenarios. All the errors of 3 scenarios are all less than the minimal error, $\mathrm{E}_{0}$. Figure 3 and Figure 4 illuminate DSFC can effectively reduce the AW delay on the condition of meeting the desired aggregation accuracy.

Under energy constraints, it is vital for sensor nodes to minimize energy consumption in radio communication to extend the lifetime of sensor networks. From the results shown in Figure 5, we argue that partial aggregation based DSFC tends to reduce the transmission of emergent packets in cluster, thus saving energy. Figure 5 shows fewer intra-cluster transmissions in scenario1, 2, and 3 consume less energy than HEED and LEACH [10], which always carry out the traditional full aggregation.

\section{Conclusion}

We have proposed a DSFC model to solve the trade-off issue between AW delay and aggregation accuracy. Simulations demonstrate DSFC model can adaptively aggregate 
partial data to reduce the AW delay on the condition of meeting the desired aggregation accuracy. The costs of energy consumption in DSFC are also very low.

\section{References}

1. LEWIS, F.L.: Wireless Sensor Networks. Smart Environments:Technologies, Protocol and Applications(2004)

2. Enachescu, M., Goel, A., Govindan, R., Motwani, R.: Scale-Free Aggregation in Sensor Networks, Theoretical Computer Science: Special Issue on Algorithmic Aspects of Wireless Sensor Networks, Vol. 344, No. 1, 11(2005)15-29

3. Younis, O., Fahmy, S.: Distributed Clustering in Ad-hoc Sensor Networks: A Hybrid, Energy-Efficient Approach. Proceedings of IEEE INFOCOM. Hong Kong(2004)

4. He, T., Stankovic, J.A., Lu, C., Abdelzaher, T.: SPEED: A stateless protocol for real-time communication in sensor networks. In Proc. of the 23rd International Confernece on Distributed Computing Systems (ICDCS-23), Providence, RI, USA,5(2003)

5. Felemban, E., Lee, C.G., Ekici, E., Boder, R., Vural, S.: Probabilistic QoS guarantee in reliability and timeliness domains in wireless sensor networks. In Proceedings of 24th Annual Joint Conference of the IEEE Computer and Communications (INFOCOM), 3(2005)2646-2657

6. Boulis, A., Ganeriwal, S., Srivastava, M.: Aggregation in Sensor Networks: An EnergyAccuracy Trade-off. Proc. of IEEE SANPA, 5(2003)1117

7. Heinzelman, W.B., et al.: An Application-Specific Protocol Architecture for Wireless Microsensor Networks. IEEE Transactions on Wireless Communications.Vol.1, No.4, 10(2002)18

8. Peng S.L., Li S.S., Liao X.K., Peng Y.X., et al.: Feedback Control with Prediction for Thread Allocation in Pipeline Architecture Web Server. In ICDCN, 2006 to be published.

9. Zeng, X., Bagrodia, R., GloMoSim, M.G.: a Library for Parallel Simulation of Large-scale Wireless Networks. In Proceedings of the 12th Workshop on Parallel and Distributed Simulations -- PADS '98, Banff, Alberta, 5(1998)

10. Younis, O., Fahmy, S.: Distributed Clustering in Ad-hoc Sensor Networks: A Hybrid, Energy-Efficient Approach.Proceedings of IEEE INFOCOM. Hong Kong, 3(2004) 